\title{
Efficient Heuristic Algorithms for Location of Charging Stations in Electric Vehicle Routing Problems
}

\author{
Gustavo GATICA ${ }^{1}$, Gerardo AHUMADA ${ }^{1}$, John Willmer ESCOBAR ${ }^{2}$, Rodrigo LINFATI $^{3 *}$ \\ ${ }^{1}$ Facultad de Ingeniería, Universidad Andres Bello, Santiago de Chile, 7591538, Chile \\ ggatica@unab.cl \\ ${ }^{2}$ Departamento de Contabilidad y Finanzas, Universidad del Valle, Cali, 760001, Colombia \\ john.wilmer.escobar@correounivalle.edu.co \\ ${ }^{3}$ Departamento de Ingeniería Industrial, Universidad del Bío-Bío, Concepción, 4030000, Chile \\ rlinfati@ubiobio.cl (*Correspondingauthor), Chile
}

\begin{abstract}
Eco-responsible transportation contributes at making a difference for companies devoted to product delivery operations. Two specific problems related to operations are the location of charging stations and the routing of electric vehicles. The first one involves locating new facilities on potential sites to minimise an objective function related to fixed and operational opening costs. The other one, electric vehicle routing problem, involves the consolidation of an electric-type fleet in order to meet a particular demand and some guidelines to optimise costs. It is determined by the distance travelled, considering the limited autonomy of the fleet, and can be restored by recharging its battery. The literature provides several solutions for locating and routing problems and contemplates restrictions that are closer to reality. However, there is an evident lack of techniques that addresses both issues simultaneously. The present article offers four solution strategies for the location of charging stations and a heuristic solution for fleet routing. The best results were obtained by applying the location strategy at the site of the client (relaxation of the VRP) to address the routing problem, but it must be considered that there are no displacements towards the recharges. Of all the other three proposals, K-means showed the best performance when locating the charging stations at the centroid of the cluster.
\end{abstract}

Keywords: Heuristics, VRP, Electric vehicle, K-means.

\section{Introduction}

'In today's economy, markets tend to be more open and competitive. The existence of an efficient, viable and environmentally friendly transportation system is a vital step towards success' (Afroditi et al. 2014). One of the strategies used by distribution companies to reduce gasoline consumption is to turn to electric vehicles. For these to transit, the arrangement of electric charging stations is necessary. The location of these facilities is an area of operations research that intends to place, at least, a new station beside the existing ones to minimise or maximise minimum one objective function (Farahani et al. 2010). Electrical vehicle routing problems (EVRP) are related to distribution logistics and difficulties in collection involving a series of customers with certain demands that must be entirely satisfied with electric freight transport vehicles, which collect the products from a warehouse. Additionally, these vehicles rely on an electric charge that decreases as they travel; hence, they require strategically located charging stations (Afroditi et al. 2014). This article proposes four strategies for the location of charging stations and one routing strategy for the fleet of electric vehicles that contemplate the reduction of the battery charge during motion. The proposed location strategies are important for the Industry 4.0. Indeed, for the smart-cities, the road infrastructure cannot grow any more, and the use of electric vehicles increases every day with the government plans to encourage and promote their use (Visser et al. al., 2017).

This paper is organized as follows: the second section includes a review of the literature on electrical location and routing problems; the third section includes four proposals for the location of charging stations and the heuristics for the subsequent routing; lastly, we include the conclusions and future studies.

\section{Literature review}

The need to propose efficient solutions to deal with recurrent industry problems, which have become evident during the literature review. In addition, it was noted that the literature only provides solutions for problems concerning the location of charging stations and electrical routing separately, whereas this article seeks to contribute with a technique that solves both problems at the same time.

\subsection{Location of charging stations}

Locating new facilities is a problem that involves several objective functions such as cost, profit, income, travel distance, service, waiting time, coverage, market shares, among others. 
There are no limitations regarding the type of location; some examples are public facilities, private facilities, military environment, business areas, warehouses and charging stations, among others (Farahani et al. 2010). For the latter, Luo et al. (2015) proposed an exact model that the owner of the electric vehicle, the owner of the recharging centre and the electric network operator benefit from. Moreover, Ghahnavieh \& Barzani (2016) proposed a mathematical model applied in Tehran (Iran) to determine the capacity and optimal location of charging stations, considering factors such as urban traffic, electric vehicle user behaviour, electronic network charging times and urban areas. Based on mobility issues, Hidalgo et al. (2016) proposed a genetic algorithm for the location of charging stations considering the actual demand of electric energy consumption, with a heterogeneous fleet of vehicles and different load behaviours. Dong et al. (2016) proposed a twophase solution; in phase 1 , they used k-means algorithm to divide a region according to the demand distribution of the market; in phase 2, they used an exact model to locate the charging stations in each region while maximising operational efficiency and convenience.

Wang et al. (2016) proposed exact models for the location of charging stations for electric buses in public transport. They also considered two location problems; one of them contemplates the vehicle's battery size, and the other one does not.

Lam et al. (2014) proposed a mathematical formulation for the problem offering four solution strategies: 1) mixed integer linear programming iterative method, 2) greedy algorithm, 3) mixed integer linear programming approach, and 4) chemical reaction optimisation. Xiong et al. (2015) proposed a mathematical model and a heuristic for the location problem at the charging stations in Singapore, considering the loading activities of the driver, the traffic and the waiting time. Most publications are recent, which demonstrates a growing interest towards studying this problem. The trend seems to lean towards proposing mathematical model strategies, the proposed approach consider four strategies, within a heuristic algorithm, for which although the use of heuristics and metaheuristics considering different constraints is also evident.

\subsection{Electric vehicle routing}

The founding article on the Vehicle Routing Problem is 'Truck Dispatching Problem' by
Dantzig and Ramser (1959); it explains how a fleet of trucks can meet the demand of a series of fuel stations from a central depot. Five years later, the VRP was formalised thanks to Clarke and Wright (1964), representing the standard VRP. Currently, VRP models are entirely different from those proposed by Dantzig and Ramser (1959) \& Clarke and Wright (1964); thus demonstrating the evolution of VRP over the years (Braekers et al. 2016). Current VRP models are much closer to reality. Other variants are customer time windows, traffic variables, different fleets of vehicles, and electric vehicles.

Braekers et al. (2016) show how research in VRP and variants has increased. In this work, it is analysed the technics to solve VRP and their variants from 2009 to 2015; where Metaheuristic algorithms outperform the rest of the proposed approaches, thanks to their effectiveness for generalisations. Exact methods are used to obtain efficient solutions for small instances Braekers et al. (2016). EVRP is an emerging area of research that arose from the need of the industry to reduce costs and to be responsible for the environment. The problem is a generalization of the traditional VRP as presented by Braekers et al. (2016), where the most investigated VRP variants are exposed. Considering several restrictions, some researchers have proposed solutions for EVRP.

Conrad \& Figliozzi (2011) proposed an exact method for the rechargeable Vehicle Routing Problem with time windows, in which the vehicles can be recharged at the customer's location while processing the service, and these can only visit at scheduled times. Some time later, Erdoğan et al. (2012) proposed a mathematical model to solve the Green Vehicle Routing Problem (GVRP), which emphasises the use of alternative energy sources, while Afroditi et al. (2014) proposed a mathematical model for the problem of vehicle routing with time windows. Also, Lin et al. (2016) proposed a mathematical model to find the optimal solution for this problem considering the effect of the battery charge on electric consumption.

Reviewing these proposals, it seems necessary to use efficient solution strategies such as heuristic or metaheuristic methods to help solving strategic and operational problems of the industry that affect large numbers of customers and this could not be achieved through exact strategies. However, the routing of electric vehicles is only part of the problem, because the road infrastructure in large 
cities does not have the capacity to grow more (Holguín-Veras et al., 2016).

\section{Solution proposal}

In this study, we attempt to strategically locate charging stations across a plane while minimising vehicular displacements using four strategies: Random Generation, Customer Location, Great Route, and K-Means. Electric vehicle routing ensures location and attempts to generate the routes vehicles must follow. For this purpose, an initial solution is obtained for the classical VRP, with the 'VRPH' library (Groër, C., 2012). Subsequently, the heuristic incorporating the particular feature of electric vehicle variant, which contemplates the autonomy of the fleet and of the charging stations. This section is divided into segments 3.1, which addresses the location of charging stations, and 3.2, for the electric vehicle routing.

\subsection{Location of charging stations}

Four algorithms were implemented for the location of the charging stations. The first one, called Random Generation, uses the number of charging stations to be located within the plane as its parameter, and then random coordinates for $X$ and $Y$ are generated within a range defined by the location of the customers. The second one is called Customer Location, in which the charging stations are affixed at the location of the customers; this means the number of charging stations will correspond to the number of customers. The third one, called Great Route, uses the number of charging stations $(N)$ required along the plane as a parameter. Then, a single route is established for all customers to locate the charging stations in the middle of the route's greatest $(N)$ arcs, where $N$ is the number of charging stations. The fourth and last algorithm, $K$-Means uses $K$ (number of charging stations to be located in the plane) as the parameter to group the customers, and thereby arrange charging stations in the centroid of each cluster.

\subsubsection{Random generation}

Random generation is the simplest location algorithm. In particular, the randomness generates certain uncertainty in the final result; Figure 1 shows a scenario where a random generation of recharge centers is carried out.

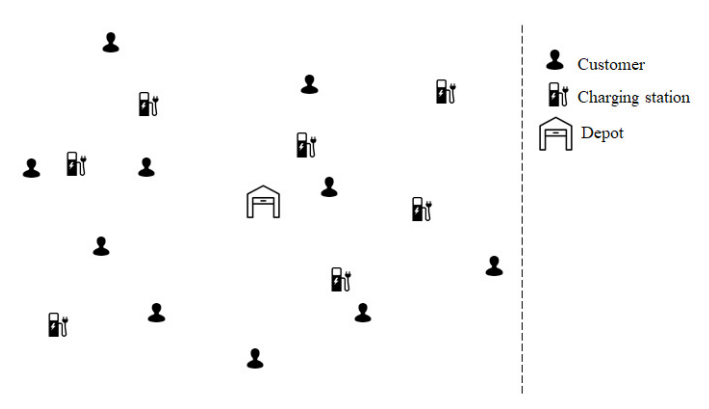

Figure 1. Random generation

\subsubsection{Location with customers}

The location of recharge centers in the location of the customers is logically the algorithm that gives the best results for performing the generated routes, because there is no displacement to recharge the vehicles. Indeed, the problem is relaxed into a traditional VRP, when the routes are performed. Figure 2 shows a scenario where each customer and the depot are a potential site to locate a recharge centre.

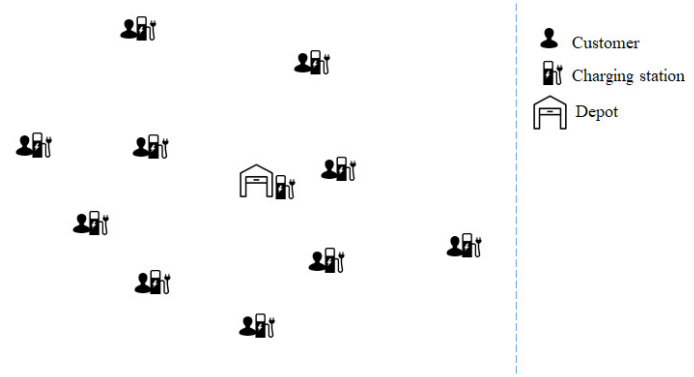

Figure 2. Location with customers

\subsubsection{Great route}

The proposed approach performs a tour for the Great Route considered as NP-Complete problem that consists in visiting exactly once a list of cities (where there is a cost to visit each city) and return to the initial node, looking for the minimum route (Hoffman et al. al., 2013). In this case, the cities are the customers, and a route is made between all the clients using the implementation of traveling salesman problem (TSP) in $\mathrm{c}++$ available at: https:/github.com/samlbest/traveling-salesman.

Once the tour is performed, the recharging centers are located in the largest $N$ arcs, where $N$ is the number of centers to be located. Figure 3 shows phase 1 of the algorithm, where the TSP tour is generated. 


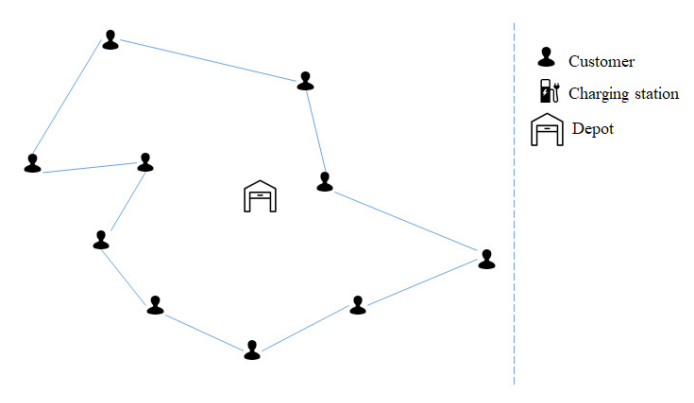

Figure 3. Great route, phase 1

Figure 4 shows the location of the recharge centers in the largest $N$ arcs $(N=4)$.

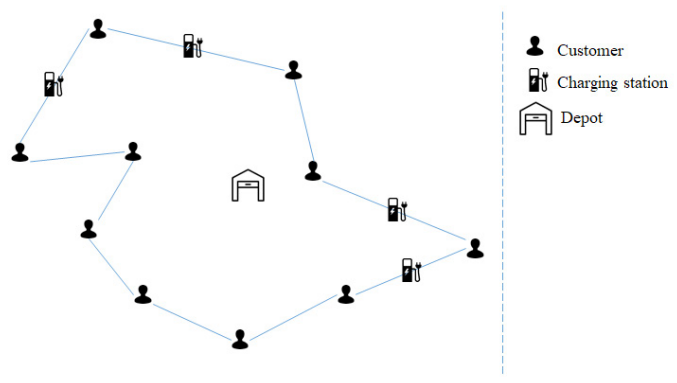

Figure 4. Great route, phase 2

Finally, Figure 5 shows the final result of the location of recharge centers.

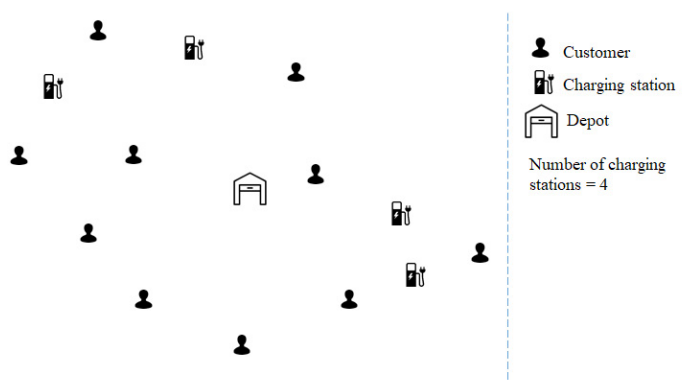

Figure 5. Great route, phase 3

\subsubsection{K-means}

The algorithm is based on the method of grouping (or cluster) K-means whose objective is to partition a set of $N$ data in $K$ groups in which each element of $N$ belongs to the group whose average value is closer. The implementation of $\mathrm{K}$-means present in the Yakmo library for $\mathrm{c}++$ is available at: www.tkl.iis.u-tokyo.ac.jp/ ynaga/ yakmo. The grouping is used to generate $\mathrm{X}$ groups of nearby customers and locate $\mathrm{X}$ recharge centers in the center of each group. Figure 6 shows phase 1 of the algorithm, where the grouping is performed $(\mathrm{K}=3)$.

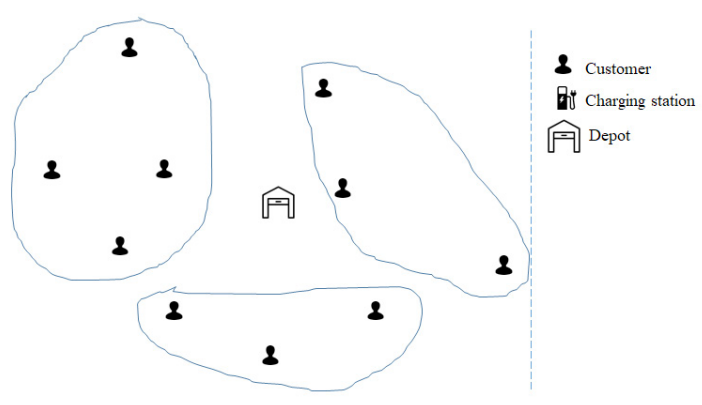

Figure 6. K-means, phase 1

Figure 7 shows the phase two of the algorithm, where the centroid of each generated cluster is calculated and a recharge center is located.

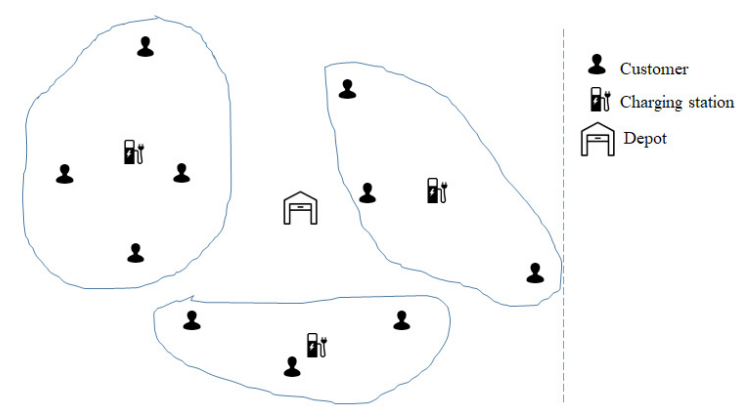

Figure 7. K-means, phase 2

Figure 8 shows the final result of the location of recharge centers.

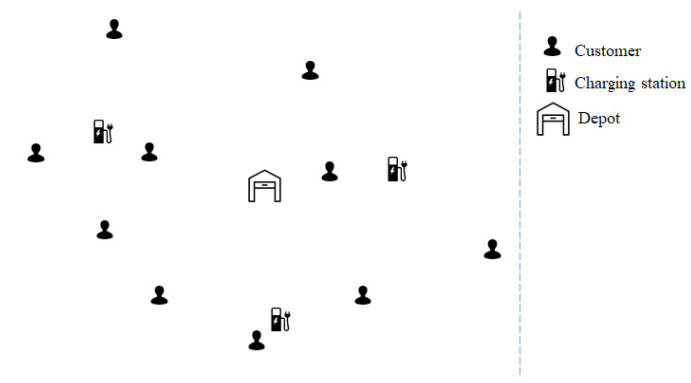

Figure 8. K-means, phase 3

\subsection{Electric vehicle routing}

For the electric vehicle routing, a four-stage procedure is followed; (I) an initial solution for the VRP is obtained using the routing library 'VRPH' proposed by Groër, C., (2012), and (II) the electrical routing algorithm is applied. In (I), the obtained solution corresponds to the classical capacitated routing problem without considering charging stations or electric vehicles. In (III) and (IV), the electric vehicle and charging station 
variants are added, considering the reduction of kilometres of autonomy when the vehicle is moving. Algorithm 1 presents the heuristics for the solution of the electric vehicle routing problem in pseudocode format.

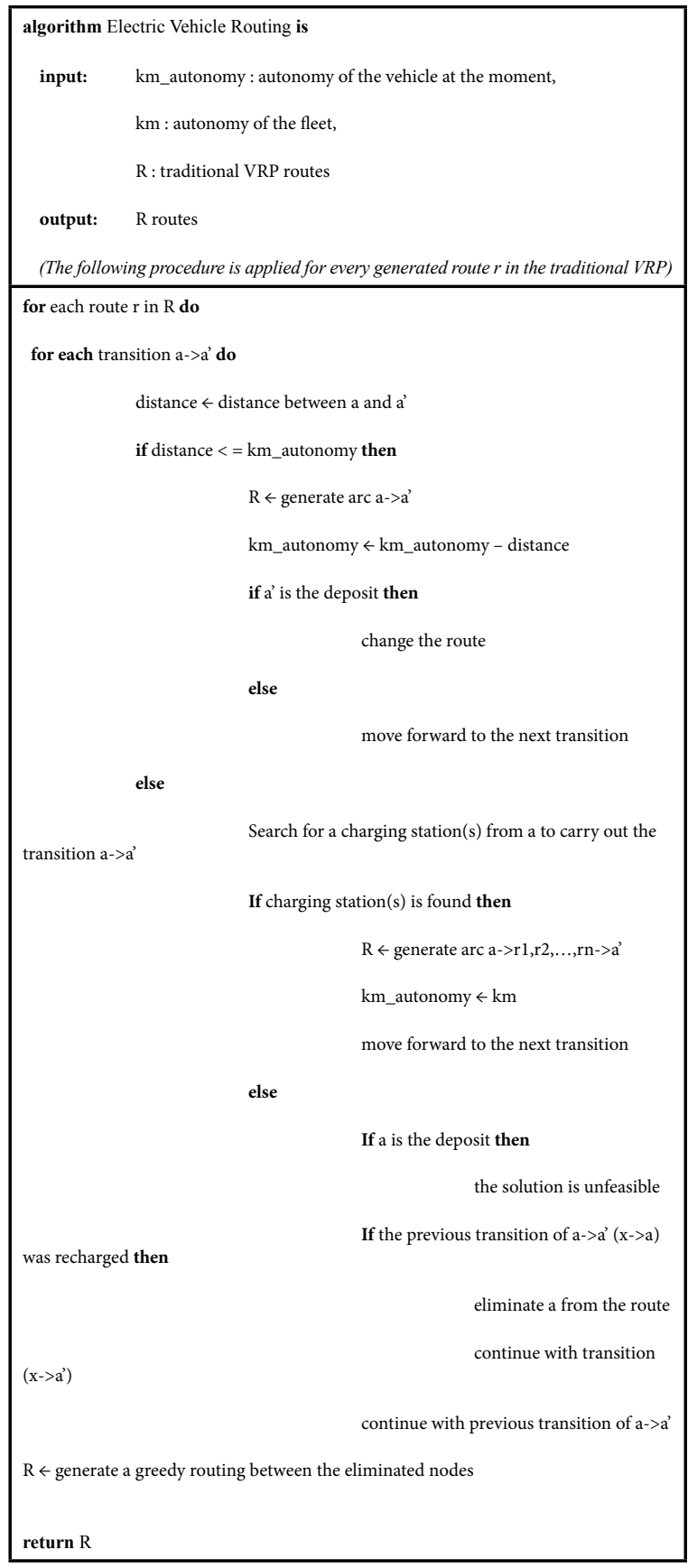

\section{Results}

This section shows the results obtained for the electric vehicle routing applying the various location strategies.

\subsection{Parameter setting}

To test the algorithms, the following settings must be established: 1.) Kilometres of autonomy, which is the distance in kilometres a vehicle can travel without recharging its battery. This parameter was set to $160 \mathrm{~km}$, based on the electric truck Smith Edison from the American company Smith Electric that we used (Smith Electric, 2016). 2.) The percentage of charging stations, which defines the number of charging stations to locate within the plan. This value multiplied by the number of customers along the route is equal to the number of charging stations, considering the whole number of the product. 3.) The location algorithm, which defines the appropriate algorithm to locate the charging stations in the plan.

\subsection{Test instances}

The VRP instances proposed by Taillard (1999), were used to test the algorithms (Universidad de Málaga, 2013). The localization of charging stations was applied to these instances using the four algorithms and, subsequently, the electric vehicle routing.

\subsection{Table of results}

Table 1 shows the results for the instances Taillard_75A.vrp., Taillard_100A.vrp. and Taillard_150A.vrp. The first column contains the name of the instance; the second column contains the number of customers; the third column contains the percentage of used charging stations; the fourth column contains the number of charging stations available; fifth to eight columns contain the objective function of the proposed approaches and of each published algorithm. Finally, the last column contains the best objective function for the VRP.

\subsection{Analysis of the results}

The analysis of the results must consider the following: the test instances apply to the traditional VRP, without taking into account the electric charging stations and the fleet; the column 'Best VRP' shown in Table 1 considers the problem as a classical VRP, and the objective function is to minimise the distance.

\subsubsection{Random generation}

This algorithm obtained the worst results with the tested instances; due to its simplicity to locate the charging stations, it does not have an elaborated procedure compared to the other three algorithms. For Taillard 75A.vrp., the best result was obtained 
with $90 \%$ of charge and worse than Best VRP by 25.18 kilometres. For Taillard_100A.vrp., the best results are obtained with $100 \%$ of charge and the worse by 54.35 kilometres compared to the best VRP. For Taillard 150A.vrp., the best results are obtained also with $100 \%$ of charge and 180.88 kilometres less than the best VRP.

\subsubsection{Location with customers}

This algorithm made the problem relax at a classic VRP and got the best results for the instances analysed, because the vehicle avoids going to a charging station to charge the battery. The best result shown in the literature was obtained for Taillard_75A.vrp. For Taillard_100A.vrp., the value obtained was 2071.43 and the best known value was only 2041.34. A closer but lower value was obtained at 30.09 kilometres. For Taillard_150A.vrp., a lower objective function was obtained at 121.88 kilometres compared to the best known. The solutions derived from the algorithm are the same or get close to the solutions shown in the literature. Considering that this location algorithm relaxes it to VRP, it can be made a balanced comparison. Figure 9 shows the layouts obtained for Taillard_75A.vrp, where the box „Customers" location“ displays the customers of the instance and, at the same time, the location of the recharge centers. Any customer location could be used to recharge the vehicle; therefore, the decision is performed at time to make the routing of the fleet. In the „Routing“ box, the final routes of the instance are displayed. The routes generated are equivalent to those of traditional VRP, with the difference that recharge points are added. In the „Total routes“ box, the order that customers are visited is presented. With a negative sign, the customers are represented where the vehicle must be recharged, for example customer 1 is represented as -2 .

\subsubsection{Great route}

The localization using the Great Route for Taillard_75A.vrp gets better results than the random generation, particularly for instances Taillard_100A.vrp and Taillard_150A.vrp. For Taillard_75A.vrp, the best result was obtained with $90 \%$ charge and worse by 35.99 kilometers compared to the best-known solution. For instance Taillard_100A.vrp, the algorithm obtains results with $100 \%$ charge and 46.87 kilometers below of the best-known solution. Finally, instance Taillard_150A.vrp shows that the best result was obtained with $100 \%$ charge and 149.17 kilometers less compared to the best-known solution.

\subsubsection{K-means}

This algorithm got better results than random generation and Great route for the three instances. For Taillard_75A.vrp., the best result was obtained with $90 \%$ charge, which was the same to the Best VRP; even though less recharges were necessary, the results were very similar. The best results for instance Taillard_100A.vrp was obtained with $100 \%$ charge and 30.09 kilometers of the distance. For Taillard_150A.vrp., the best result was obtained with $100 \%$ charge and 121.88 fewer kilometers than the best known. Figure 10 shows the layouts obtained for Taillard_75A. vrp, where the "k-means" box displays the clients grouped under the $\mathrm{k}$-means algorithm $(\mathrm{k}=68)$. The customers are grouped with the objective of locating recharge centers that can supply a set of nodes. After grouping, a recharge center is located in each centroid of the cluster. Then, the "routing" box presents the final routes of the instance. Finally, the "total routes" box shows, the order that customers are visited. Indeed, with a negative sign, the customers are represented where the vehicle must be recharged, for example customer 1 is represented as -2 .

\section{Conclusion and future research}

\subsection{Location of charging stations}

The contribution created by the proposal of this solution makes possible to extrapolate the location of installations, plants, and storehouses. It is relevant to mention that in a real scenario, the enterprises that incorporate policies for the use of electric vehicles will aim for a fewer number of charging stations to minimise costs. Then, the analysis in this respect must be conducted by examinating the different proposed scenarios in which the value of charging stations varies.

Among the four proposals for the location of charging stations, we conclude the following: with regards to Random Generation, we can say it is the only core algorithm based on randomness, 
Table 1. Instance results, where Best VRP is the best result found in the literature

\begin{tabular}{|c|c|c|c|c|c|c|c|c|}
\hline \multirow[b]{2}{*}{ Instance } & \multirow[b]{2}{*}{$\begin{array}{c}\mathrm{N}^{\circ} \text { of } \\
\text { customers }\end{array}$} & \multirow[b]{2}{*}{$\begin{array}{c}\% \text { of } \\
\text { recharges }\end{array}$} & \multirow[b]{2}{*}{$\begin{array}{c}\text { № } \\
\text { Recharges }\end{array}$} & \multicolumn{5}{|c|}{ Objective function } \\
\hline & & & & $\begin{array}{l}\text { Random } \\
\text { generation }\end{array}$ & $\begin{array}{c}\text { Customers' } \\
\text { location }\end{array}$ & $\begin{array}{l}\text { Great } \\
\text { route }\end{array}$ & K-means & Best VRP \\
\hline $\begin{array}{l}\text { Taillard_75A. } \\
\text { vrp }\end{array}$ & $\begin{array}{l}75 \\
75 \\
75 \\
75 \\
75 \\
75 \\
75 \\
75 \\
75 \\
75\end{array}$ & $\begin{array}{l}10 \% \\
20 \% \\
30 \% \\
40 \% \\
50 \% \\
60 \% \\
70 \% \\
80 \% \\
90 \% \\
100 \%\end{array}$ & $\begin{array}{l}7 \\
15 \\
22 \\
30 \\
38 \\
45 \\
53 \\
60 \\
68 \\
76\end{array}$ & $\begin{array}{l}1715.58 \\
1673.14 \\
1670.2 \\
1672.42 \\
1668.66 \\
1665.78 \\
1650.73 \\
1659.53 \\
1643.54 \\
1648.43\end{array}$ & $\begin{array}{l}1618.36 \\
1618.36 \\
1618.36 \\
1618.36 \\
1618.36 \\
1618.36 \\
1618.36 \\
1618.36 \\
1618.36 \\
1618.36\end{array}$ & $\begin{array}{l}1693.63 \\
1724.31 \\
1710.76 \\
1717.43 \\
1678.81 \\
1669.94 \\
1665.33 \\
1657.04 \\
1654.35 \\
1654.35\end{array}$ & $\begin{array}{l}1744.31 \\
1668.02 \\
1658.45 \\
1653.39 \\
1636.03 \\
1629.08 \\
1621.12 \\
1622.68 \\
1618.36 \\
1618.36\end{array}$ & 1618.36 \\
\hline $\begin{array}{l}\text { Taillard_100A. } \\
\text { vrp }\end{array}$ & $\begin{array}{l}100 \\
100 \\
100 \\
100 \\
100 \\
100 \\
100 \\
100 \\
100 \\
100\end{array}$ & $\begin{array}{l}10 \% \\
20 \% \\
30 \% \\
40 \% \\
50 \% \\
60 \% \\
70 \% \\
80 \% \\
90 \% \\
100 \%\end{array}$ & $\begin{array}{l}10 \\
20 \\
30 \\
40 \\
50 \\
60 \\
70 \\
80 \\
90 \\
101\end{array}$ & $\begin{array}{l}2231.44 \\
2174.16 \\
2130.18 \\
2126.88 \\
2127.68 \\
2109.43 \\
2103.85 \\
2113.29 \\
2113.7 \\
2095.69\end{array}$ & $\begin{array}{l}2071.43 \\
2071.43 \\
2071.43 \\
2071.43 \\
2071.43 \\
2071.43 \\
2071.43 \\
2071.43 \\
2071.43 \\
2071.43\end{array}$ & $\begin{array}{l}2734.51 \\
2228.55 \\
2195.18 \\
2164.18 \\
2136.69 \\
2108.31 \\
2094.42 \\
2094.42 \\
2094.42 \\
2088.21\end{array}$ & $\begin{array}{l}2208.44 \\
2175.64 \\
2124.75 \\
2094.7 \\
2087.74 \\
2079.86 \\
2073.26 \\
2073.26 \\
2072.81 \\
2071.43\end{array}$ & 2041.34 \\
\hline $\begin{array}{l}\text { Taillard_150A. } \\
\text { vrp }\end{array}$ & $\begin{array}{l}150 \\
150 \\
150 \\
150 \\
150 \\
150 \\
150 \\
150 \\
150 \\
150\end{array}$ & $\begin{array}{l}10 \% \\
20 \% \\
30 \% \\
40 \% \\
50 \% \\
60 \% \\
70 \% \\
80 \% \\
90 \% \\
100 \%\end{array}$ & $\begin{array}{l}15 \\
30 \\
45 \\
60 \\
75 \\
90 \\
105 \\
120 \\
135 \\
151\end{array}$ & $\begin{array}{l}3452.09 \\
3383.6 \\
3330.97 \\
3287.49 \\
3256.19 \\
3249.94 \\
3249.92 \\
3243.37 \\
3257.37 \\
3236.11\end{array}$ & $\begin{array}{l}3177.11 \\
3177.11 \\
3177.11 \\
3177.11 \\
3177.11 \\
3177.11 \\
3177.11 \\
3177.11 \\
3177.11 \\
3177.11\end{array}$ & $\begin{array}{l}3475.92 \\
3327.2 \\
3256.15 \\
3237.88 \\
3212.19 \\
3217.72 \\
3211.64 \\
3209.65 \\
3209.65 \\
3204.4\end{array}$ & $\begin{array}{l}3277.74 \\
3243.06 \\
3244.59 \\
3212.7 \\
3201.12 \\
3182.53 \\
3181.81 \\
3180.92 \\
3177.79 \\
3177.11\end{array}$ & 3055.23 \\
\hline
\end{tabular}

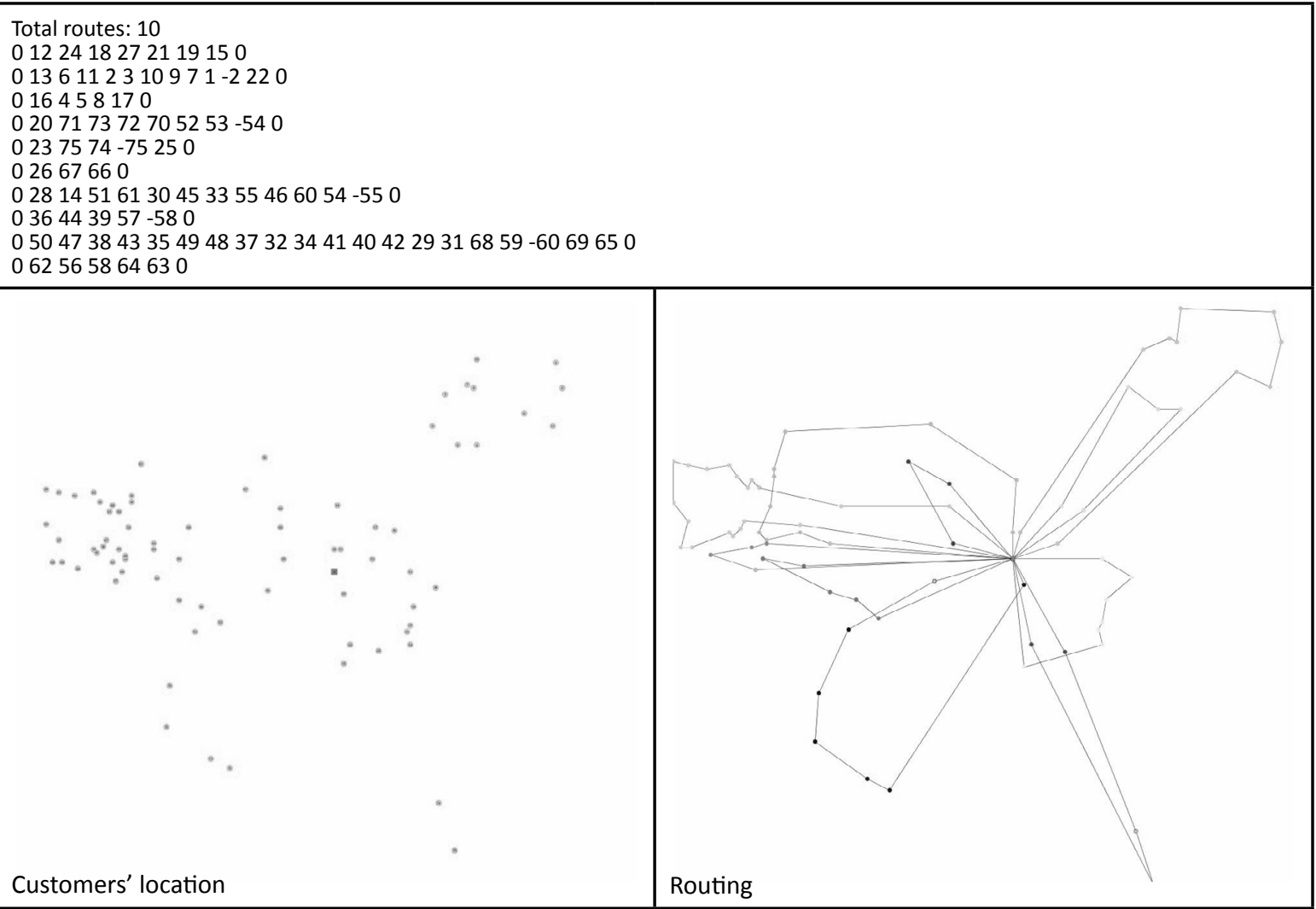

Figure 9. Customers' location for Taillard_75A.vrp., where the customers are represented by positive numbers, the charging stations by negative numbers, and the deposit is 0 


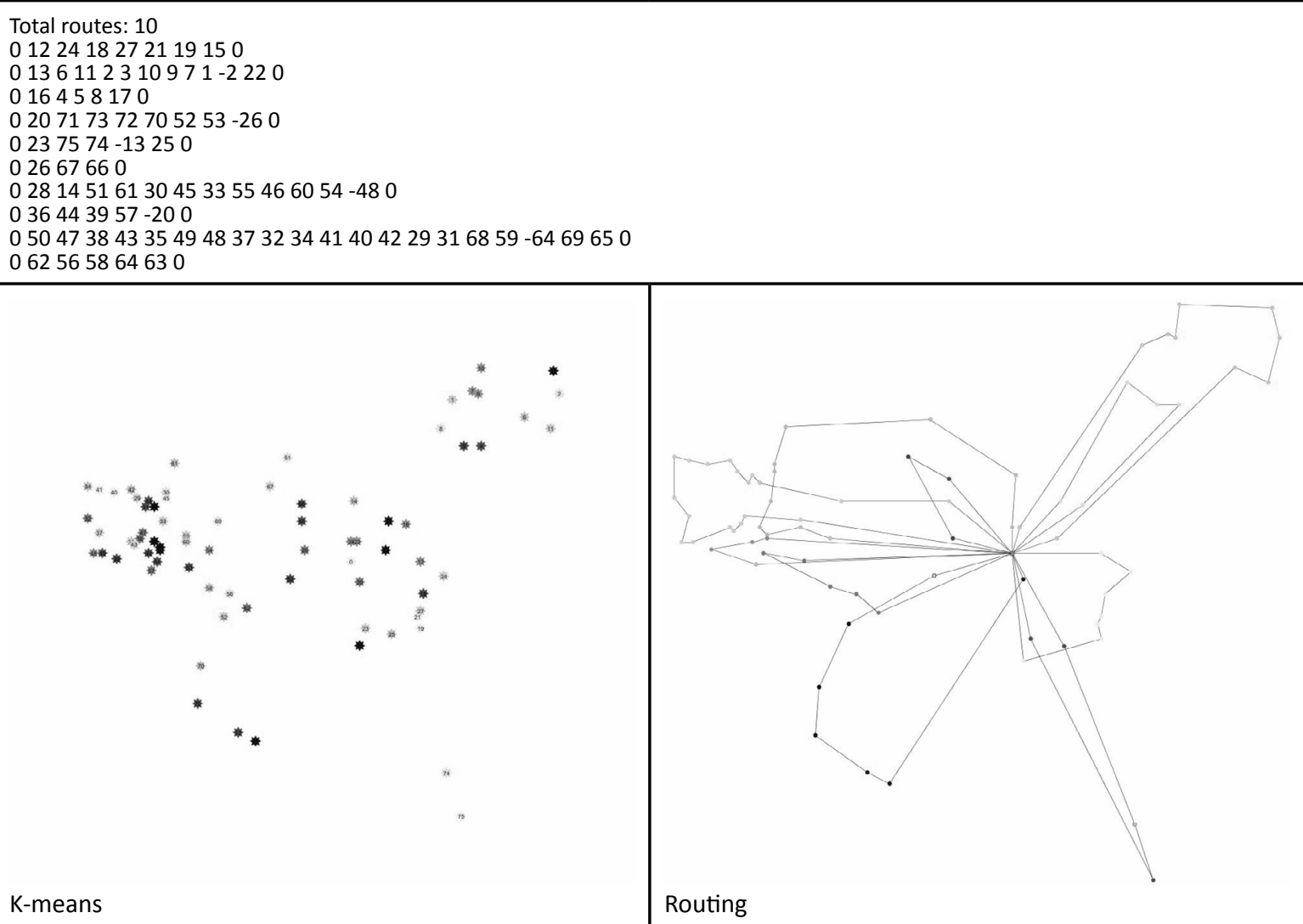

Figure 10. K-means 90\% for Taillard 75A.vrp., where the customers are represented by positive numbers, the charging stations by negative numbers, and the deposit is 0

this is why the results obtained with this algorithm were worse than those obtained with the other proposals. With regards to the location of the customers, we can conclude that this solution proposal relaxes the EVRP to a VRP; since there is no displacement towards a charging station, the charging process happens at the location of the customers. The solutions obtained are better compared to the other proposals, although subdued to a real scenario.

This project could be unfeasible due to the location of the charging stations at the customers' location. The purpose of the Great Route proposal is to locate charging stations between greater distances and customers so that it is feasible for the electric vehicle to cover wider ranges. The results obtained with this algorithm were, in most cases, superior to the random generation but not better than those of the k-means proposal and of the location of the customers. Finally, the purpose of the k-means algorithm is to locate the charging stations to supply a close group of customers, and it is carried out with the creation of groups and, subsequently, the setting of the location of a charging centre in the centroid of the group. The results obtained with this algorithm were better than those obtained with Random Generation and Great Route but inferior to the results obtained with the customers' location.

\subsection{Electric vehicle routing}

The main contribution exposed in the article is the generation of routes considering the electric vehicle fleet, using a heuristic-efficient procedure and with the help of a specialised library such as VRPH. The results obtained using the location of the customers strategy (relaxation to VRP) can be improved via some additional route interchange or procedures, in order to achieve results even closer to the best known in the literature.

\section{Acknowledgements}

This work has been partially supported by CONICYT FONDECYT by grant 11150370 , FONDEF IT17M10012 and the "Grupo de Logística y Transporte" at the Universidad del Bío-Bío.. This support is gratefully acknowledged. 


\section{REFERENCES}

1. Afroditi, A., Anagnostopoulou, A., Maria, B., Sotirios, T., Eleftherios, S. \& Dimitrios, M. (2014). Electric Vehicle Routing Problem with Industry Constraints: Trends and Insights for Future Research, Transportation Research Procedia, 3, 452-459.

2. Braekers, K., Kris, B., Katrien, R. \& Van Nieuwenhuyse, I. (2015). The vehicle routing problem: State of the art classification and review, Computers \& Industrial Engineering, 99, 300-313.

3. Clarke, G. \& Wright, J. W. (1964). Scheduling of Vehicles from a Central Depot to a Number of Delivery Points, Operations Research, 12(4), 568-581.

4. Conrad, R. G. \& Figliozzi, M. A. (2011). The recharging vehicle routing problem. In Proceedings of the 2011 Industrial Engineering Research Conference, Reno, Nevada, USA.

5. Dantzig, G. B. \& Ramser, J. H. (1959). The Truck Dispatching Problem, Management Science, 6(1), 80-91.

6. Dong, Y., Qian, S., Liu, J., Zhang, L. \& Zhang, K. (2016). Optimal placement of charging stations for electric taxis in urban area with profit maximization. In 2016 $17^{\text {th }}$ IEEE/ACIS International Conference on Software Engineering, Artificial Intelligence, Networking and Parallel/ Distributed Computing (SNPD). $<$ https://doi. org/10.1109/snpd.2016.7515897>.

7. Erdoğan, S., Sevgi, E. \& Elise, M.-H. (2012). A Green Vehicle Routing Problem, Transportation Research Part E: Logistics and Transportation Review, 48(1), 100-114.

8. Farahani, R. Z., SteadieSeifi, M. \& Asgari, N. (2010). Multiple criteria facility location problems: A survey, Applied Mathematical Modelling, 34(7), 1689-1709.

9. GitHub. (2018). samlbest/traveling-salesman [online]. Accessed on 19 Feb. 2018. Available at: $<$ https://github.com/samlbest/travelingsalesman $>$.
10. Groër, C. (2012). Vehicle Routing Problem Heuristics. URL: <http://www.coin-or.org/ projects/VRPH.xml>.

11. Hidalgo, P. A. L., Lopez Hidalgo, P. A., Ostendorp, M. \& Lienkamp, M. (2016). Optimizing the charging station placement by considering the user's charging behavior. In 2016 IEEE International Energy Conference (ENERGYCON). < <ttps://doi.org/10.1109/ energycon.2016.77513920>.

12. Holguín-Veras, J., Encarnación, T., GonzálezCalderón, C. A., Winebrake, J., Wang, C., Kyle, S. \& Yoshizaki, H. (2016). Direct impacts of off-hour deliveries on urban freight emissions, Transportation Research Part D: Transport and Environment.

13. Lam, A. Y. S., Leung, Y.-W. \& Chu, X. (2014). Electric Vehicle Charging Station Placement: Formulation, Complexity, and Solutions, IEEE Transactions on Smart Grid, 5(6), 2846-2856.

14. Lin, J., Zhou, W.\& Wolfson, O. (2016). Electric Vehicle Routing Problem, Transportation Research Procedia, 12, 508-521.

15. Luo, C., Huang, Y. F. \& Gupta, V. (2017). Placement of EV Charging Stations Balancing Benefits Among Multiple Entities, IEEE Transactions on Smart Grid, 8(2), 759768. doi: 10.1109/TSG.2015.2508740.

16. Rajabi-Ghahnavieh, A. \& Sadeghi-Barzani, P. (2016). Optimal Zonal Fast Charging Station Placement Considering Urban Traffic Circulation, IEEE Transactions on Vehicular Technology, 66(1), 45-56.

17. Smith Electric. (2016). Smith Electric: Canada's Choice for Auto Electrical Parts \& Service. Smith Electric (Febrero 2017). Sitio web: <http://www.smithelectric.ca/>.

18. Taillard, É. D. (1999). A heuristic column generation method for the heterogeneous fleet VRP, RAIRO-Operations Research, 33(1), 1-14.

19. Universidad de Málaga. (2013). The Vehicle 
Routing Problem (VRP). Networking and Emerging Optimization (Febrero 2017). Sitio web: <http://neo.lcc.uma.es/vrp/>.

20. Visser, J., Allen, J., Browne, M., HolguinVeras, J. \& Ng, J. (2017). Light Commercial Vehicles (LCVs) in urban areas, revisited. In $10^{\text {th }}$ International Conference on City Logistics, 14 June 2017, Phuket, Thailand.

21. Wang, X., Yuen, C., Hassan, N. U., An, N. \& Wu, W. (2017). Electric vehicle charging station placement for urban public bus systems, IEEE Transactions on Intelligent Transportation Systems, 18(1), 128-139.
22. Xiong, Y., Gan, J., An, B., Miao, C. \& Bazzan, A. L. (2015). Optimal electric vehicle charging station placement. In Proceedings of the $24^{\text {th }}$ International Joint Conference on Artificial Intelligence (IJCAI), Beunos Aires, Argentina (pp. 2662-2668).

23. Yoshinaga, N. (2016). yakmo - C++ implementation of robust, efficient alternative $k$-means clustering [online]. Accessed on 19 Feb. 2018. Available at: <http://www.tkl. iis.u-tokyo.ac.jp/ ynaga/yakmo/> . 